\title{
Theoretical Evidence of Solvent-Mediated Excited-State Dynamics in a Functionalized
} Iron Sensitizer

Pápai, Mátyás Imre; Abedi, Mostafa; Levi, Gianluca; Biasin, Elisa; Nielsen, Martin Meedom; Møller, Klaus Braagaard

Published in:

Journal of Physical Chemistry C

Link to article, DOI:

10.1021/acs.jpcc.8b10768

Publication date:

2019

Document Version

Peer reviewed version

Link back to DTU Orbit

Citation (APA):

Pápai, M. I., Abedi, M., Levi, G., Biasin, E., Nielsen, M. M., \& Møller, K. B. (2019). Theoretical Evidence of Solvent-Mediated Excited-State Dynamics in a Functionalized Iron Sensitizer. Journal of Physical Chemistry $C$, 123(4), 2056-2065. https://doi.org/10.1021/acs.jpcc.8b10768

\section{General rights}

Copyright and moral rights for the publications made accessible in the public portal are retained by the authors and/or other copyright owners and it is a condition of accessing publications that users recognise and abide by the legal requirements associated with these rights.

- Users may download and print one copy of any publication from the public portal for the purpose of private study or research.

- You may not further distribute the material or use it for any profit-making activity or commercial gain

- You may freely distribute the URL identifying the publication in the public portal 
Subscriber access provided by DTU Library

\title{
C: Energy Conversion and Storage; Energy and Charge Transport
}

\author{
Theoretical Evidence of Solvent-Mediated Excited- \\ State Dynamics in a Functionalized Iron Sensitizer \\ Mátyás Pápai, Mostafa Abedi, Gianluca Levi, Elisa Biasin, \\ Martin Meedom Nielsen, and Klaus Braagaard Møller
}

J. Phys. Chem. C, Just Accepted Manuscript • DOI: 10.1021/acs.jpcc.8b10768 • Publication Date (Web): 03 Jan 2019

Downloaded from http://pubs.acs.org on January 5, 2019

\section{Just Accepted}

"Just Accepted" manuscripts have been peer-reviewed and accepted for publication. They are posted online prior to technical editing, formatting for publication and author proofing. The American Chemical Society provides "Just Accepted" as a service to the research community to expedite the dissemination of scientific material as soon as possible after acceptance. "Just Accepted" manuscripts appear in full in PDF format accompanied by an HTML abstract. "Just Accepted" manuscripts have been fully peer reviewed, but should not be considered the official version of record. They are citable by the Digital Object Identifier (DOI®). "Just Accepted" is an optional service offered to authors. Therefore, the "Just Accepted" Web site may not include all articles that will be published in the journal. After a manuscript is technically edited and formatted, it will be removed from the "Just Accepted" Web site and published as an ASAP article. Note that technical editing may introduce minor changes to the manuscript text and/or graphics which could affect content, and all legal disclaimers and ethical guidelines that apply to the journal pertain. ACS cannot be held responsible for errors or consequences arising from the use of information contained in these "Just Accepted" manuscripts. 


\title{
Theoretical Evidence of Solvent-Mediated
}

\section{Excited-State Dynamics in a Functionalized Iron Sensitizer}

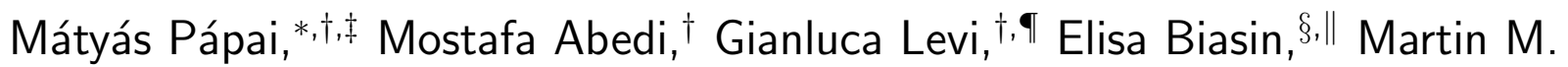 \\ Nielsen, ${ }^{\S}$ and Klaus B. Møller ${ }^{\dagger}$ \\ †Department of Chemistry, Technical University of Denmark, DK-2800, Kongens Lyngby, \\ Denmark \\ ‡Wigner Research Centre for Physics, Hungarian Academy of Sciences, P.O. Box 49, \\ H-1525 Budapest, Hungary \\ ФFaculty of Physical Sciences, University of Iceland, 107 Reykjavik, Iceland \\ $\S$ Department of Physics, Technical University of Denmark, Fysikvej 30\%, DK-2800 \\ Kongens Lyngby, Denmark \\ ॥PULSE Institute, SLAC National Accelerator Laboratory, Menlo Park, CA 94025, USA \\ E-mail: papai@kemi.dtu.dk
}




\begin{abstract}
The solvent-mediated excited-state dynamics of the COOH-functionalized Fe-carbene photosensitizer $\left[\mathrm{Fe}(\mathrm{bmicp})_{2}\right]^{2+}(\mathrm{bmicp}=2,6$-bis $(3-$ methyl-imidazole-1-ylidine $)$-4-carboxypyridine) is studied by time-dependent density functional theory (TD-DFT), as well as classical and quantum dynamics simulations. We demonstrate the crucial role of the polar acetonitrile solvent in stabilizing the MLCT states of the investigated molecule using the conductor polarizable continuum (CPCM) model. This leads to dynamics that avoid sub-ps back electron transfer to the metal, and an exceptionally long-lived ${ }^{1}$ MLCT state that does not undergo sub-ps ${ }^{1} \mathrm{MLCT} \rightarrow{ }^{3} \mathrm{MLCT}$ intersystem crossing, as it is energetically isolated. We identify two components of the excited-state solvent reorganization process: an initial rotation ( $\sim 300 \mathrm{fs})$ and diffusional dynamics within the local cage surrounding the rotated solvent molecule $(\sim 2 \mathrm{ps})$. Finally, it is found that the relaxation of the solvent only slightly affects the excited-state population dynamics of $\left[\mathrm{Fe}(\mathrm{bmicp})_{2}\right]^{2+}$.
\end{abstract}

\title{
1 Introduction
}

Excited-state dynamics in transition metal complexes ${ }^{1-3}$ (TMCs) are ubiquitous and a key to develop advanced technologies, solar energy conversion, ${ }^{4}$ photocatalysis, ${ }^{5,6}$ molecular data storage, ${ }^{7,8}$ just to name a few. Among these intriguing molecular systems, Fe-N-heterocyclic carbenes $^{9}$ (NHCs) received recently special attention owing to their great potential as cheap, Earth-abundant photosensitizers. This is due to their relatively long-lived (ps) photoactive metal-to-ligand charge transfer (MLCT) states that can be exploited, for instance, to inject the photoexcited electron into the conduction band of a semiconductor. This injection process into $\mathrm{TiO}_{2}$ was indeed observed with $92 \%$ yield in the $\left[\mathrm{Fe}(\text { bmicp })_{2}\right]^{2+}($ bmicp $=$ 2,6-bis(3-methyl-imidazole-1-ylidine)-4-carboxy-pyridine) complex, however the majority of these electrons were found to undergo fast ( $<10 \mathrm{~ns}$ ) recombination with cations, thus preventing efficient photocurrent generation. ${ }^{10}$ In order to overcome such drawbacks, it is inevitable 
to gain a thorough mechanistic understanding of the underlying photophysics. As photorelaxation processes, such as internal conversion (IC), intersystem crossing (ISC), and vibrational dynamics (VD), occur on the fs-ps timescale, ultrafast pump-probe techniques are needed to explore these molecular transformations. This field, born with optical ${ }^{11}$ pump-probe experiments and afterwards expanded to the use of X-rays ${ }^{12,13}$ and electrons ${ }^{14}$ as probe pulses, is going through a very robust technolgical evolution, exemplified by high-harmonic generation (HHG) and X-ray free electron lasers (XFELs). These advancements enable high-resolution spatial and temporal investigations of electronic and structural photoinduced dynamics in molecular systems. However, the data analysis of these ultrafast spectroscopic and scattering measurements can be remarkably complex and cumbersome, making the interpretation problematic. This obstacle can be overcome by the application of theoretical approaches, which can lead to complementary results and even to the design of new experiments.

Theory offers two conceptually different approaches to simulate excited-state processes: nonadiabatic molecular dynamics ${ }^{3,15}$ (MD) and quantum wavepacket dynamics (QD). ${ }^{16}$ The major methodological difference is the description of nuclear motion, which is classical for the former, with quantum effects only partially accounted for, e.g., by hops between adiabatic surfaces, as in trajectory surface hopping; while for the latter, it is fully quantum mechanical, i.e., based on the solution of the nuclear time-dependent Schrödinger equation. However, full QD simulations are heavily restricted by their exponential scaling with the number of nuclear degrees of freedom (DoF); due to this, model Hamiltonians are developed, in which only the modes most relevant for the dynamics are included. In contrast to "on-the-fly" surface hopping, for these Hamiltonians, potential energy surfaces (PESs) and interstate couplings do not have to be recomputed throughout the dynamics, but only once, along the selected modes, preceding the actual QD simulation. As a matter of fact, while surfacehopping studies of TMCs have been restricted to sub-ps timescales ${ }^{17-19}$ due to the high cost of computing ab initio electronic properties at each time step, longer timescales have been accessible only through recent QD simulations. ${ }^{19-25}$ 
Numerous chemical and biological excited-state processes occur in solution, which is also the medium utilized in the vast majority of pump-probe experiments. Therefore, it is crucial to assess solvent effects on the excited-state dynamics, also when comparing simulations to experiments. This is evidenced by the observation of solvent-dependent photorelaxation decay in transition-metal-based coordination compounds including the diplatinum $\left[\mathrm{Pt}_{2}\left(\mathrm{P}_{2} \mathrm{O}_{5} \mathrm{H}_{2}\right)_{4}\right]^{4-}(\mathrm{PtPOP})$ complex ${ }^{26-28}$ and $\left[\mathrm{Fe}\left(2,2^{\prime} \text {-bipyridine }\right)_{2}(\mathrm{CN})_{4}\right]^{2-} .{ }^{29}$ In the case of surface-hopping MD, the solvent can be readily incorporated in the simulations in the framework of electrostatic- or polarizable-embedding hybrid quantum mechanincs/molecular mechanics (QM/MM). ${ }^{3,17,30,31}$ For QD, however, simulations on TMCs have so far been carried out in vacuum, the only exceptions being the works of Daniel et al. on Re(I) complexes, ${ }^{21,24,25}$ in which the solvent has been included using a variant of polarizable continuum models (PCMs), the conductor-like screening model (COSMO). We mention that recently a mixed quantum-classical method has been proposed, applying different approaches to couple the excited-state solute-solvent dynamics, ${ }^{32}$ albeit for the internal conversion of a relatively small organic molecule, thymine, not TMCs. In the present work, we assess along similar lines the impact of solvent effects on the simulated excited-state population dynamics of $\left[\mathrm{Fe}(\mathrm{bmicp})_{2}\right]^{2+}(\mathbf{1}$, Scheme 1, top). Importantly, this COOH-functionalized Fe(II)-NHC is the complex in which the above-mentioned electron injection was experimentally observed. Moreover, it was found in acetonitrile (ACN) to exhibit twice as long MLCT lifetime (18 ps, Scheme 1, bottom), compared to the one of its parent complex, $\left[\mathrm{Fe}(\mathrm{bmip})_{2}\right]^{2+}(\mathbf{2})(\mathrm{bmip}=$ 2,6-bis(3-methyl-imidazole-1-ylidine)-4-pyridine), as measured by transient absorption spectroscopy. ${ }^{9,10,33,34}$ 


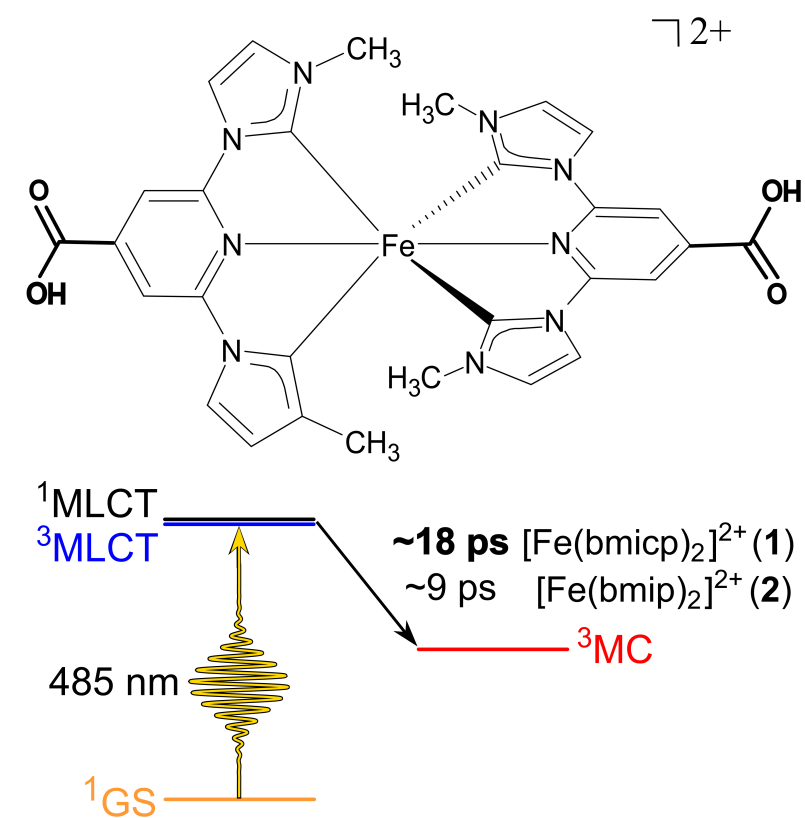

Scheme 1: Graphical illustration of the molecular structure (top) and photophysics (bottom) of $\left[\mathrm{Fe}(\text { bmicp })_{2}\right]^{2+}(\mathbf{1})$ and the parent complex, $\left[\mathrm{Fe}(\text { bmip })_{2}\right]^{2+}(\mathbf{2}$, which has $\mathrm{H}$ atoms instead of the COOH groups). 9 ps and 18 ps are the MLCT lifetimes of $\left[\mathrm{Fe}(\mathrm{bmip})_{2}\right]^{2+}$ and $\left[\mathrm{Fe}(\text { bmicp })_{2}\right]^{2+}$, respectively, as determined by transient absorption spectroscopy experiments performed in acetonitrile. ${ }^{10,33}$

\section{Theoretical and Computational Methods}

\subsection{Quantum Mechanical Calculations and Simulations}

\subsubsection{Spin-Vibronic Hamiltonian}

The spin-vibronic Hamiltonian ${ }^{20-24}$ is an extension of the widely-adopted vibronic-coupling Hamiltonian $^{35,36}$ by the introduction of spin-orbit coupling (SOC) between different spin manifolds.

In the present work, we employ a diabatic vibronic-coupling Hamiltonian that is expressed as:

$$
\boldsymbol{H}=\left(T_{N}+V_{0}\right) \mathbf{1}+\boldsymbol{W}
$$

here $T_{N}$ and $V_{0}$ are the kinetic and potential energy operators of the ground-state har- 
monic oscillator, $\mathbf{1}$ is unit matrix, and $\boldsymbol{W}$ is the potential coupling matrix. We expand the coupling matrix up to second-order:

$$
W=W^{(0)}+W^{(1)}+W^{(2)}
$$

$\boldsymbol{W}^{(0)}$ is a diagonal matrix, whose elements are the excitation energies of the electronic states at the reference point, in the present case the Franck-Condon (FC) geometry:

$$
W_{i}^{(0)}=E_{i}
$$

The diagonal elements of the $\boldsymbol{W}^{(\mathbf{1})}$ matrix are related to the forces acting on the excitedstate surfaces:

$$
W_{i}^{(1)}=\sum_{\alpha}^{f} \kappa_{i, \nu_{\alpha}} Q_{\nu_{\alpha}},
$$

here $\kappa_{i, \nu_{\alpha}}$ are linear diagonal expansion coefficients for electronic state $i$ and nuclear degree of freedom (DoF) $\nu_{\alpha}, Q_{\nu_{\alpha}}$ is the mass-frequency weighted dimensionless normal coordinate of normal mode $\nu_{\alpha}$, and $f$ is the total number of modes included in the model.

The off-diagonal elements of the $\boldsymbol{W}^{(\mathbf{1})}$ matrix represent the interstate nonadiabatic couplings:

$$
W_{i j}^{(1)}=\sum_{\alpha}^{f} \lambda_{i j, \nu_{\alpha}} Q_{\nu_{\alpha}}
$$

where $\lambda_{i j, \nu_{\alpha}}$ are the linear off-diagonal coupling coefficients between electronic states $i$ and $j(i \neq j)$ along mode $\nu_{\alpha}$.

The $\boldsymbol{W}^{(2)}$ matrix is taken to be diagonal; its elements describe the differences between ground- and excited-state vibrational frequencies:

$$
\begin{gathered}
W_{i}^{(2)}=\frac{1}{2} \sum_{\alpha}^{f} \gamma_{i, \nu_{\alpha}} Q_{\nu_{\alpha}}^{2}, \\
6 \\
\text { ACS Paragon Plus Environment }
\end{gathered}
$$


where $\gamma_{i, \nu_{\alpha}}$ are the second-order diagonal expansion coefficients. Second-order off-diagonal coefficients and higher-order terms can be important for anharmonic effects; as the computed excited-state potentials are harmonic (see Figures S1-S16 in the Supporting Information, SI), these parameters were not included in our model.

The $\kappa_{i, \nu_{\alpha}}, \lambda_{i j, \nu_{\alpha}}$, and $\gamma_{i, \nu_{\alpha}}$ values were determined by fitting the parameters, such that the obtained adiabatic potential energy surfaces (PESs), resulting from the diagonalization of the diabatic Hamiltonian, are in best agreement with those computed by electronic structure calculations (in the present case, time-dependent density functional theory, TD-DFT). This method is known as diabatization by ansatz. ${ }^{37,38}$ In the fit, 292 parameters were optimized to 2184 TD-DFT points, in overall (thus, the risk of overfitting is minimized). The parameters are given in Tables S1-S14. The fit is carried out separately for singlet and triplet states, whose adiabatic surfaces are shown in Figures S1-S16, the diabatic potentials are depicted in Figures S17-20. The coupling between the singlet and triplet manifold is introduced via the inclusion of SOC matrix elements, leading to the spin-vibronic Hamiltonian:

$$
\boldsymbol{H}=\left(T_{N}+V_{0}\right) \mathbf{1}+\boldsymbol{W}+\boldsymbol{S},
$$

where $\boldsymbol{S}$ is the SOC matrix.

The four nuclear DoF included in the applied Hamiltonians of the investigated $\left[\mathrm{Fe}(\mathrm{bmicp})_{2}\right]^{2+}$ complex (1) were adapted from our recent QD study of the parent complex, $\left[\mathrm{Fe}(\mathrm{bmip})_{2}\right]^{2+}$ (2) ${ }^{22}$ These include two low-frequency tuning ( $\nu_{6}$ and $\nu_{46}$, breathing-like modes) and coupling modes ( $\nu_{15}$ and $\nu_{35}$, antisymmetric stretching modes), identified by the magnitude of linear coupling constants $\kappa_{i, \nu_{\alpha}}$ (tuning modes, here $i$ refer to MC states) and $\lambda_{i j, \nu_{\alpha}}$ (coupling modes). For the animation of the four modes, see the MPG files in the SI. We included all singlet and triplet electronic states that are lower-lying or in the energetic vicinity of the optically-active ${ }^{1} \mathrm{MLCT}$ at the FC geometry. This led to the inclusion of 8 singlet excited states, $6{ }^{1}$ MLCTs and $2{ }^{1} \mathrm{MCs}$, 11 triplet excited states, $7{ }^{3} \mathrm{MLCTs}$ and $4{ }^{3} \mathrm{MCs}$; accounting for the three triplet components, overall 41 excited states. 


\subsubsection{Electronic Structure Calculations}

The ground-state $\left({ }^{1} \mathrm{GS}\right)$ structure of $\mathbf{1}$ was fully optimized and the normal modes were calculated at the B3LYP*39/TZVP 40 level of theory. No imaginary frequencies were found, confirming the optimized geometry is a true minimum of the PES. Subsequently, TDB3LYP*/TZVP computations were carried out at the ${ }^{1} \mathrm{GS}$ equilibrium geometry and on structures distorted along the 4 normal modes. The TD-B3LYP* exchange-correlation functional has recently been found to correctly describe the excited-state energetics of transition metal complexes. ${ }^{41-43}$ Here we performed two sets of calculations: in vacuum and acetonitrile $(\mathrm{ACN})$. In the latter case, the solvent was modeled by the conductor polarizable continuum model $^{44}$ (QM/CPCM, where QM refers to the quantum mechanical treatment of the solute) for the ${ }^{1}$ GS geometry optimization and normal modes. In excited-state PCM models, the charges of the solvent have two different contributions: orientational ("slow", governed by the static dielectric constant) and electronic ("fast", governed by the optical dielectric constant). We here consider the so-called nonequilibrium regime, ${ }^{45}$ in which the orientational solvent charges are relaxed to the ground state, while electronic charges are relaxed to the excited-state electron density of the solute. This approach is widely-used for electronic absorption and the calculation of excited-state PESs. ${ }^{46,47}$ Nonequilibrium TD-DFT/CPCM calculations were performed within the linear response (LR) formalism. ${ }^{48}$ All these electronic structure calculations were performed using the Gaussian09 ${ }^{49}$ program package.

The SOC matrix elements were calculated at the FC geometry utilizing the perturbative approach of Ziegler et al.. ${ }^{50}$ These calculations were carried out at the B3LYP*/TZP level using the zeroth-order regular approximation (ZORA), ${ }^{51}$ in vacuum and modeling the solvent environment by COSMO, ${ }^{52}$ as implemented in the ADF2016 program. ${ }^{53}$ The Gaussian and ADF-computed electronic states were checked for consistency. 


\subsubsection{Quantum Dynamics Simulations}

The nuclear time-dependent Schrödinger equation was solved using the Multiconfiguration Time-Dependent Hartee $\left(\mathrm{MCTDH}^{54}\right)$ ansatz:

$$
\Psi\left(Q_{1}, \ldots, Q_{f}, t\right)=\sum_{j_{1}=1}^{n_{1}} \ldots \sum_{j_{f}=1}^{n_{f}} A_{j_{1} \ldots j_{f}}(t) \varphi_{j_{1}}^{(1)}\left(Q_{1}, t\right) \ldots \varphi_{j_{f}}^{(f)}\left(Q_{f}, t\right)
$$

as implemented in the the Heidelberg code (version 8.4). ${ }^{55}$ Here, $A_{j_{1} \ldots j_{f}}(t)$ and $\varphi_{j_{1}}^{(1)}\left(Q_{1}, t\right) \ldots \varphi_{j_{f}}^{(f)}\left(Q_{f}, t\right)$ are the sets of variatonally-optimized time-dependent expansion coefficients and basis functions, i.e., SPFs, respectively, for $f$ number of nuclear DoF. The SPFs are then further expanded in a time-independent primitive basis set, in the present case, into harmonic oscillator $(\mathrm{HO})$ basis functions $\left(\chi_{k}^{\nu_{\alpha}}\right)$ :

$$
\varphi_{j}^{\nu_{\alpha}}=\sum_{k=1}^{N_{\nu_{\alpha}}} a_{k j}^{\left(\nu_{\alpha}\right)} \chi_{k}^{\nu_{\alpha}}\left(Q_{\nu_{\alpha}}\right)
$$

The ground state wavepacket, built using one-dimensional harmonic oscillator functions with zero initial momentum, was projected to the excited-state surface of the optically-active ${ }^{1}$ MLCT state $\left(\mathrm{S}_{5}\right)$. In Tables $\mathrm{S} 18$ and $\mathrm{S} 19$, we report the computation details of the QD simulations utilizing the 4-mode/42-state vacuum and nonequlibrium-CPCM Hamiltonians, respectively. These parameters ensured converged dynamics for the full 10 ps of the excitedstate QD simulations.

\subsection{Classical Molecular Dynamics Simulations}

All classical MD simulations were carried out with periodic boundary conditions (PBCs), using the multistep RESPA integrator ${ }^{56}$ with a time step of $1 \mathrm{fs}$, as implemented in the Desmond-v4.5 program package. ${ }^{57}$ The OPLS-AA ${ }^{58}$ and the three-site force field by Guàrdia et $a l .{ }^{59}$ were applied for the solute and the solvent, respectively. 


\subsubsection{Equilibrium MD Simulations}

For these simulations, we followed the methodology applied in our recent study ${ }^{60}$ that contrasted classical and QM/MM simulations on transition metal complexes, prototypical for excited-state dynamics. The first step of this procedure is the optimization of the ${ }^{1} \mathrm{GS}$ and lowest-lying ${ }^{3}$ MLCT state of 1 at the B3LYP*/TZVP level, in vacuum, followed by the calculation of partial atomic charges at the optimized geometry (data presented in Tables S16 and S17). For the latter, we utilized the CHELPG method, ${ }^{61}$ which we recently found to lead to equilibrium radial distribution functions (RDFs) in good agreement with those simulated by QM/MM. ${ }^{60}$ The next step is the solvation of the molecule in a $50 \AA$ x $50 \AA$ x $50 \AA$ cubic box of ACN molecules, applying two $\mathrm{Cl}^{-}$anions to nutralize the complex. We employed the protocol of Desmond to equilibrate the solvated system at constant NVT, applying a Berendsen thermostat. ${ }^{62}$ This includes the following steps: i) 100 ps Brownian dynamics (NVT) at $T=10 \mathrm{~K}$ with restraints on solute heavy atoms, ii) $12 \mathrm{ps}$ NVT at $T=10 \mathrm{~K}$ with restraints on solute heavy atoms, and iii) $24 \mathrm{ps}$ NVT at $T=300 \mathrm{~K}$ and no restraints. This led to equilibrated ${ }^{1} \mathrm{GS}$ and ${ }^{3}$ MLCT trajectories. Subsequently, a 2 ns production run in the NVT ensemble $(T=300 \mathrm{~K})$, utilizing a Nosé-Hoover thermostat, ${ }^{63}$ was carried out. The atoms of the solute and the $\mathrm{Cl}^{-}$anions (which were placed away from the complex) were restrained during the production using a harmonic potential with a force constant of $1000 \mathrm{kcal} \times \mathrm{mol}^{-1} \times \AA^{-2}$. By this, we could avoid the additional effort of reparametrizing the solute, while at the same time, maintaining the accuracy of the simulated RDFs, as shown in ref. 60. The trajectory was recorded at each 50 fs of the simulation, which was then used to calculate the equilibrium ${ }^{1} \mathrm{GS}$ and ${ }^{3} \mathrm{MLCT}$ solute-solvent RDFs.

Solvent configurations extracted from these trajectories were used for calculations, in which both the solute and solvent are treated by TD-DFT (explicit-solvent QM). As TDDFT calculations on the full MD box are not feasible, we truncated the molecular system to the solute plus the first Fe- $\mathrm{N}(\mathrm{ACN})$ and $\mathrm{H}(\mathrm{COOH})-\mathrm{N}(\mathrm{ACN})$ coordination shell (see Figure $\mathrm{S} 27)$; this leads to the inclusion of $32 \mathrm{ACN}$ molecules, on average. Test computations 


\section{Results and Discussion}

Figure 1 depicts the excited-state singlet/triplet MLCT and metal-centered (MC, originating from $d \rightarrow d$ transitions) potential energy curves of 1 along the principal mode for the dynamics, accounting for the most significant structural distortions (breathing-like normal mode $\nu_{6}$ in the SI). Comparison of the potentials calculated in vacuum (Figure 1a, see also Table S2) with those of $\mathbf{2}^{22}$ (reported in Table S15) reveals that the COOH groups stabilize 
the MLCT states by $0.30-0.35 \mathrm{eV}$, in agreement with previous experimental and computational results, ${ }^{10,34,64}$ while the MCs are slightly destabilized by $0.03-0.10 \mathrm{eV}$. Figure $2 \mathrm{~b}$ (Table S9) presents the nonequilibrium QM/CPCM-calculated excited-state potentials. The results display a $0.15-0.19 \mathrm{eV}$ red shift and a $0.04-0.07 \mathrm{eV}$ blue shift for MLCT and MC states, respectively, relative to the potentials calculated in vacuum, shown in Figure 1a. In particular, the energy of the initially excited (optically bright) ${ }^{1}$ MLCT state (black dashed line in Figure 1), at the FC geometry drops from $2.81 \mathrm{eV}$ (vacuum) to $2.62 \mathrm{eV}$ (QM/CPCM), which means a significant improvement of the agreement with respect to the maximum of the experimental MLCT absorption band located at $2.38 \mathrm{eV}(520 \mathrm{~nm}) \cdot{ }^{10,34}$ This solvent effect is highlighted in Figure 1c, in which the vacuum and QM/CPCM-calculated excited-state potentials are shown in the same panel, but for clarity, only for a selection of representative states of each character and multiplicity. The above-observed stabilization of MLCT states can be understood by basic electrostatics: the charge transfer leads to an increased dipole moment in the excited state, and thus increased interaction with the dipoles of the polar solvent. Interestingly, this MLCT stabilization by the solvent does not occur for the parent complex, 2. We therefore attribute the MLCT red shift, observed for 1, to the increased charge transfer character of its MLCT states, caused by the COOHsubstitution. This is confirmed by the significantly larger change in dipole moment between the ground state and the lowest-lying ${ }^{3} \mathrm{MLCT}$ obtained for $\mathbf{1}$ than for $\mathbf{2}(0.38 \mathrm{D}$ and $0.02 \mathrm{D}$, respectively.)

In the following, we assess the influence of the solvent on the photorelaxation of $\mathbf{1}$ by QD simulations utilizing vacuum and QM/CPCM-derived spin-vibronic Hamiltonians. We present the resulting excited-state population dynamics in Figure 2. In vacuum, we observe an ultrafast deactivation of the photoexcited ${ }^{1}$ MLCT state via internal conversion into the ${ }^{1} \mathrm{MC}$ with an exponential time constant of $\sim 150$ fs (Figure $2 \mathrm{a}$ ). The reason for this very fast deactivation is that the ${ }^{1} \mathrm{MLCT} /{ }^{1} \mathrm{MC}$ intersection occurs at the $\mathrm{FC}$ geometry, as is clear from Figure 1a. The ${ }^{1} \mathrm{MLCT} \rightarrow{ }^{1} \mathrm{MC}$ IC is followed by population transfer into ${ }^{3} \mathrm{MLCT}$ 

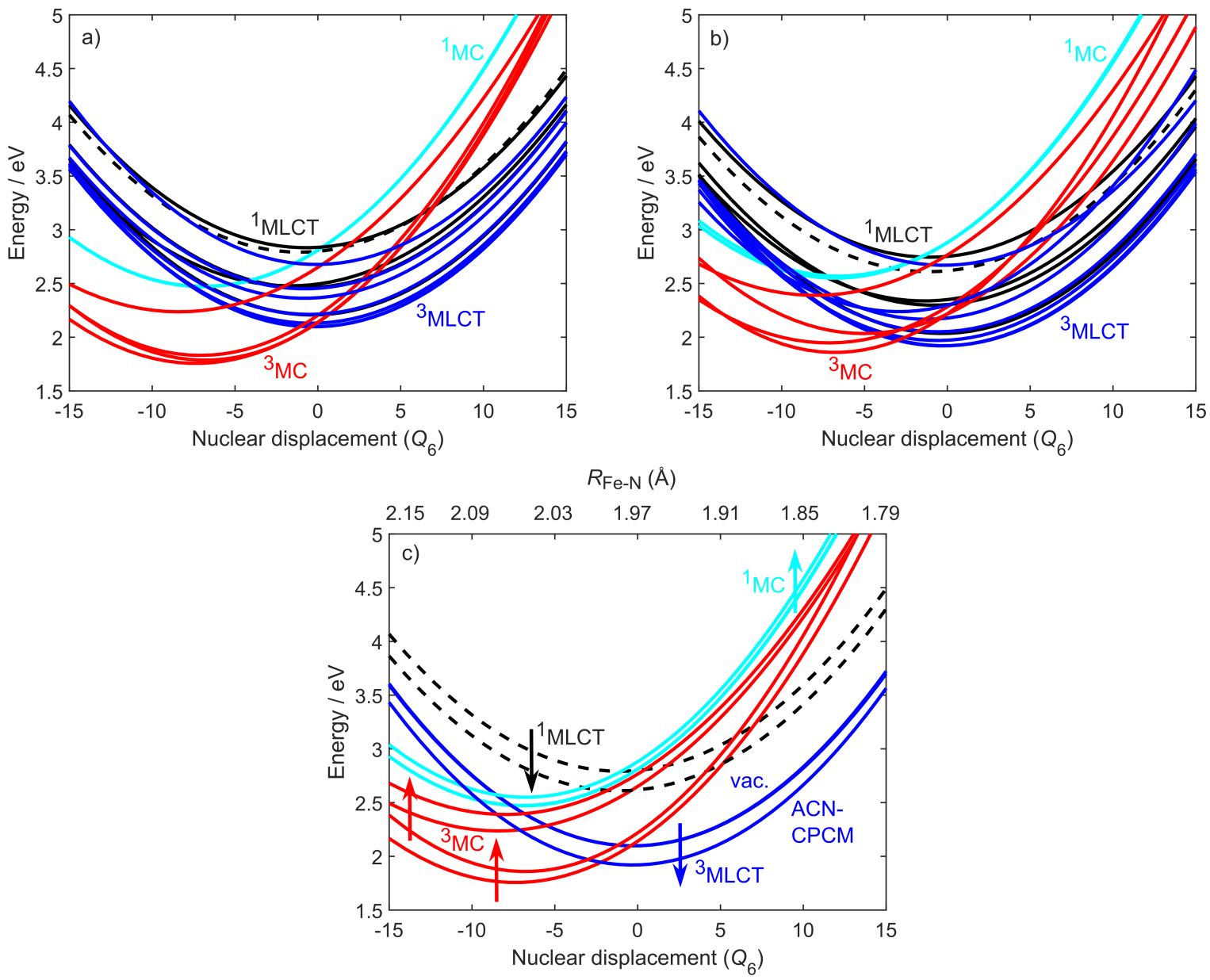

Figure 1: Diabatic excited-state potentials of $\left[\mathrm{Fe}(\mathrm{bmicp})_{2}\right]^{2+}$ along the $\nu_{6}$ breathing-like vibrational mode, dominant for the dynamics, in a) vacuum and b) acetonitrile, simulated by nonequilibrium QM/CPCM. The dashed line represents the potential of the opticallybright ${ }^{1}$ MLCT state to which the electronic excitation occurs at the Franck-Condon (FC) geometry. c) depicts the excited-state vacuum and QM/CPCM-calculated potentials of a selection of the electronic states. Here, arrows pointing down and up denote the solvent effect on the corresponding potentials, stabilization and destabilization, respectively. Nuclear displacements are given in dimensionless mass-frequency scaled normal coordinates. The average distance between the $\mathrm{Fe}$ and the $\mathrm{N}$ of the pyridine ring $\left(R_{\mathrm{Fe}-\mathrm{N}}\right)$ for the CPCMcalculated geometries is also shown.

and ${ }^{3} \mathrm{MC}$ states via intersystem crossing, occurring on a slower timescale, characterized by an exponential $\sim 750$ fs time constant. This deactivation mechanism is inconsistent with the experimental observations, ${ }^{10,34}$ as it involves the back transfer of the photoexcited electron to the Fe on the subpicosecond timescale. Crucially, such short timescales would not allow 
electron injection into a semiconductor with high yield (e.g., 92\%, as reported in ref. 10), as the injection process was found to occur in $\sim 3$ ps. This discrepancy highlights the importance of excited-state solvent effects in $\mathbf{1}$. In Figure 2b, we present the excited-state populations obtained from the QD simulation using the nonequilibrium QM/CPCM-derived Hamiltonian. This figure shows that in ACN, the sub-ps IC into the ${ }^{1} \mathrm{MC}$ is completely quenched and the simulated ${ }^{1}$ MLCT lifetime ( $>10 \mathrm{ps}$ ) is enhanced by two orders of magnitude, relative to the one obtained in vacuum. This drastic change in the dynamics is attributed to the dislocation of the ${ }^{1} \mathrm{MLCT} /{ }^{1} \mathrm{MC}$ intersection from the $\mathrm{FC}$ point due to the red shift of MLCT states caused by the interaction with the solvent. The inclusion of the solvent at the QM/CPCM level leads to results in agreement with the experiments; ${ }^{10,34}$ importantly, no significant back transfer to the metal is observed during the initial dynamics. Interestingly, in contrast to the widely-adopted excited-state dynamics of TMCs, ${ }^{1,65-68}$ the QM/CPCM-QD does not exhibit any ultrafast (sub-ps) ISC between the ${ }^{1}$ MLCT and ${ }^{3}$ MLCT states. This is because all lowerlying ${ }^{3}$ MLCT states are energetically well-separated from the photoexcited ${ }^{1}$ MLCT state at the FC geometry, as is clear from Figure $1 \mathrm{~b}$. The absence of ${ }^{1} \mathrm{MLCT} \rightarrow{ }^{3} \mathrm{MLCT}$ ISC has the important consequence that our QD simulations exceptionally identify the photoexcited ${ }^{1}$ MLCT as the long-lived excited state of 1.

As shown above, the excited-state nonequilibrium QM/CPCM treatment of the solute leads to a significant improvement of the simulated dynamics. This is attributed to the fact that QM/CPCM correctly accounts for the electrostatic stabilization of MLCT states. However, this approach neglects the orientational ("slow") response of the solvent, which can modulate the excited-state energetics and consequently, can affect the population dynamics. Nevertheless, this modulation would only occur if the solvent reorientation is fast enough, in comparison with the population dynamics (several ps, in the present case, see Figure 2b). In order to investigate the timescales of the solvent dynamics, we perform classical MD simulations in ACN. As a first step, we carry out equilibrium MD simulations in the ${ }^{1} \mathrm{GS}$ and lowest-lying ${ }^{3} \mathrm{MLCT}$ state of $\mathbf{1}$. Note that the choice of this ${ }^{3}$ MLCT instead 

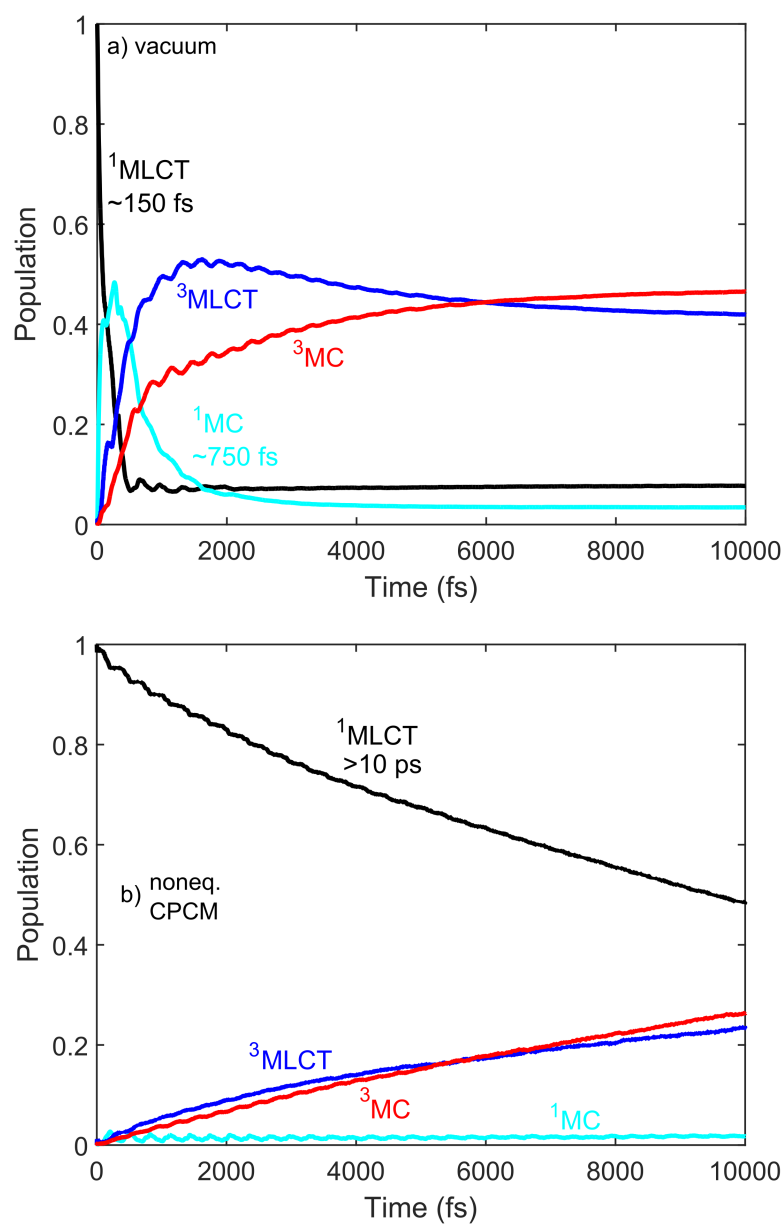

Figure 2: Diabatic relaxation kinetics of $\left[\mathrm{Fe}(\text { bmicp })_{2}\right]^{2+}$ following photoexcitation into the optically bright ${ }^{1}$ MLCT state, as obtained from QD simulations performed in a) vacuum and b) acetonitrile (nonequilibrium QM/CPCM). The excited-state lifetimes were obtained by exponential fits.

of the photoexcited ${ }^{1} \mathrm{MLCT}$ as the excited state is due to a technical reason: it enables geometry optimization by unrestricted DFT, while this was not possible for the ${ }^{1}$ MLCT by TD-DFT. However, this does not introduce any inconsistencies, as differences in the relaxed structure and excited-state density for these two states is negligible. This is confirmed by the comparison of the computed partial charges and dipole moment for the two states.

In Figure 3, we characterize the equilibrium solvation structure in the ${ }^{1} \mathrm{GS}$ and ${ }^{3} \mathrm{MLCT}$ states by the obtained pairwise Fe-N(ACN) RDFs and the resulting ratio of $n_{\mathrm{Fe}-\mathrm{N}(\mathrm{ACN})}$ and $n_{\mathrm{Fe}-\mathrm{CH}_{3}(\mathrm{ACN})}$ (inset). The ${ }^{1} \mathrm{GS}_{-}{ }^{3} \mathrm{MLCT}$ changes seen in the first two peaks of the RDFs and the $n_{\mathrm{Fe}-\mathrm{N}(\mathrm{ACN})} / n_{\mathrm{Fe}-\mathrm{CH}_{3}(\mathrm{ACN})}$ ratio clearly show evidence of reorganization of the first 
coordination shell in the excited state. ACN molecules (two on average, as determined by $n_{\mathrm{Fe}-\mathrm{N}(\mathrm{ACN})}$ corresponding to the first valley in the ${ }^{3} \mathrm{MLCT}$ Fe-N(ACN) RDF) located between the two bmicp ligands, rotate such that their nitrogen end is oriented towards the Fe center; this is reflected in the increase of the $n_{\mathrm{Fe}-\mathrm{N}(\mathrm{ACN})} / n_{\mathrm{Fe}-\mathrm{CH}_{3}(\mathrm{ACN})}$ ratio in the excited state. The solvent structural changes in the excited state are dominated by reorganization of the first coordination shell, the change in the number of solvent molecules in the first shell is negligible. This result is in agreement with the fact that electronic transitions to MLCT states remove electronic density from the Fe, making the metallic center more positive and hence more prone to attract the negatively charged nitrogen moiety of the solvent. We use the obtained equilibrium RDFs as references for subsequent out-of-equilibrium MD simulations, aimed to probe the excited-state solvent dynamics. ${ }^{69,70}$ These simulations yield a $~ 300$ fs timescale for the above-reported rotation of ACN towards the Fe center, identified by the increase of $n_{\mathrm{Fe}-\mathrm{N}(\mathrm{ACN})}$ and $n_{\mathrm{Fe}-\mathrm{N}(\mathrm{ACN})} / n_{\mathrm{Fe}-\mathrm{CH}_{3}(\mathrm{ACN})}$ as a function of time (Figures $\mathrm{S} 23$ and S24). Additionally, a significantly slower $\sim 2$ ps kinetic component also occurs; this is attributed to the diffusional dynamics of the cage of solvent molecules around the rotated ones, characterized by large-amplitude oscillations in $n_{\mathrm{Fe}-\mathrm{N}(\mathrm{ACN})}, n_{\mathrm{Fe}-\mathrm{CH}_{3}(\mathrm{ACN})}$ and their ratio (Figures S23-S26). These results show that the solvent relaxation occurs on a substantially faster timescale than the simulated population dynamics in $\mathrm{ACN}$, shown in Figure 2b. It is, therefore, possible that the solvent relaxation can affect the population dynamics.

We address the effect of solvent relaxation on the excited-state energetics by explicitsolvent QM (TD-DFT) computations, carried out on 150 uncorrelated (separated by 10 ps in the corresponding equilibrated trajectory) ${ }^{1} \mathrm{GS}$ and ${ }^{3} \mathrm{MLCT}$ MD snapshots. Here, we have performed two sets of calculations: one using the solute only (vac) and another which also includes explicit solvent molecules (sol), as obtained from the MD simulations, leading to the $\Delta E=E_{\mathrm{sol}}-E_{\mathrm{vac}}$ solvent shifts of excitation energies. In Figure 4, we depict the histograms and Gaussian-fitted normalized probability distribution functions (PDFs) of $\Delta E$ for the optically-active ${ }^{1} \mathrm{MLCT}$, as well as the lowest-lying ${ }^{1} \mathrm{MLCT},{ }^{3} \mathrm{MLCT},{ }^{3} \mathrm{MC}$ 


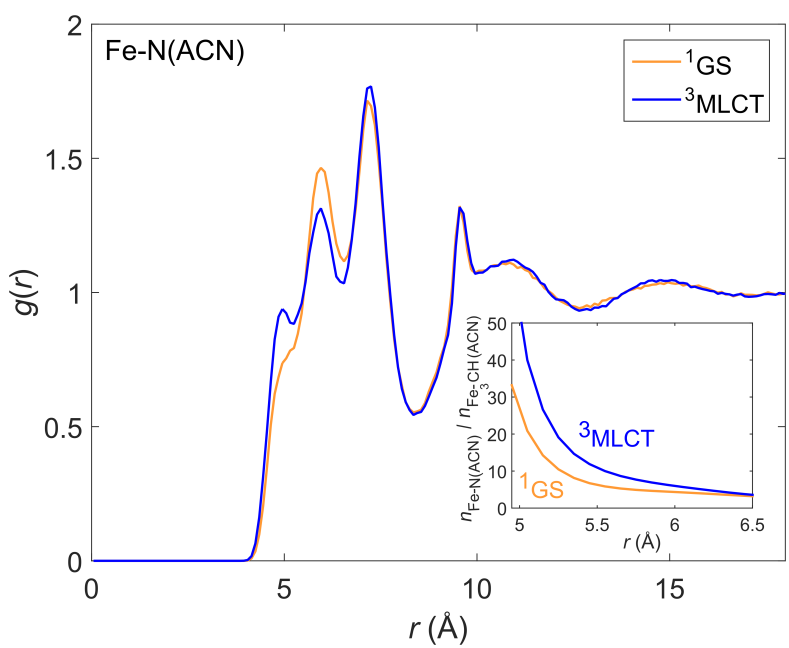

Figure 3: Equilibrium pairwise Fe-N(ACN) RDFs obtained from the MD simulations utilizing ground (orange) and ${ }^{3} \mathrm{MLCT}$ excited-state (blue) solute structure and partial atomic charges. A bin size of $0.1 \AA$ was used for the radial sampling. The inset shows the $n_{\mathrm{Fe}-\mathrm{N}(\mathrm{ACN})} / n_{\mathrm{Fe}-\mathrm{CH}_{3}(\mathrm{ACN})}$ ratio calculated from the corresponding ${ }^{1} \mathrm{GS}$ and ${ }^{3} \mathrm{MLCT}$ RDFs.

states, as obtained from TD-DFT calculations using ${ }^{1} \mathrm{GS}$ (orange) and ${ }^{3} \mathrm{MLCT}$ (blue) solvent configurations. We also illustrate, for comparison, the corresponding $\Delta E$ values calculated by $\mathrm{QM} / \mathrm{CPCM}\left(\Delta E_{\mathrm{CPCM}}\right.$, vertical dashed lines in Figure 4). As discussed previously, in nonequilibrium-PCM solvation, the orientational charges of the solvent are relaxed to the ground-state density of the solute; this is appropriate for contrasting explicit-solvent QM calculations utilizing ${ }^{1} \mathrm{GS}$ solvent configurations (orange histograms in Figure 4). However, this is no longer the case when excited-state solvent configurations are used in explicitsolvent QM (blue histograms in Figure 4); therefore, for contrasting these calculations, we employed equilibrium-PCM solvation within the state-specific (SS) formalism, ${ }^{71}$ in which both orientational and electronic solvent charges are relaxed to the excited-state solute density. The results presented in Figure 4 show a remarkable agreement between nonequilibrium $\Delta E_{\mathrm{CPCM}}$ and the maximum of the corresponding ${ }^{1} \mathrm{GS}$ probability distributions; a similarly high accuracy of PCM methods compared to explicit-solvent QM calculations has been also recently reported for the $\pi \pi^{*}$ and $n \pi^{*}$ transitions of small organic molecules. ${ }^{46}$ Based on the presented explicit-solvent QM calculations, the solvent relaxation has a rather different effect on the energetics of different excited states. As seen in Figure 4, the excited-state 
distributions (blue) for the lowest-lying ${ }^{1} \mathrm{MLCT}$ and ${ }^{3} \mathrm{MLCT}$ states (Figures 4b and 4c) are shifted by $-0.15 \mathrm{eV}$, relative to the corresponding ground-state (orange) distributions. On the other hand, this shift is $-0.06 \mathrm{eV}$ for the optically-active ${ }^{1} \mathrm{MLCT}$, and only $-0.02 \mathrm{eV}$ for the lowest-lying ${ }^{3} \mathrm{MC}$ (for ${ }^{1} \mathrm{MC}$ states, this shift is even smaller). These results lead to the important conclusion that the solvent reorganization can modulate the excited-state energetics, which is a key factor for the dynamics. Moreover, the red shift caused by the solvent relaxation is reproduced by the equilibrium QM/CPCM calculations, even if deviations up to $0.06 \mathrm{eV}$, relative to the explicit-solvent QM shifts are observed.

Finally, we assess how the population dynamics is affected by the solvent relaxation. For this purpose, we have carried out a series of QD simulations, in which the QM/CPCMcalculated potentials are shifted according to different excited-state solvent configurations. The shift is obtained by the comparison of explicit-solvent QM-calculated $\Delta E$ values, for the 150 snapshots of the excited-state MD trajectory, to the nonequilibrium QM/CPCMcalculated solvent stabilization (noneq. $\Delta E_{\mathrm{CPCM}}$ in Figure 4), for states with different multiplicity and character (optically-active ${ }^{1} \mathrm{MLCT},{ }^{1} \mathrm{MLCT},{ }^{3} \mathrm{MLCT},{ }^{1} \mathrm{MC}$, and ${ }^{3} \mathrm{MC}$ ).

In Figure 5, we present the ${ }^{1}$ MLCT populations extracted from the 150 QD simulations, each corresponding to a particular excited-state solvent configuration, as well as their average, contrasted to those obtained from the QD simulations using the gas-phase and nonequilibrium QM/CPCM Hamiltonians. These results show that different excited-state solvent environments can lead to very different ${ }^{1}$ MLCT decays that range from the nearly vacuum limit to negligible decay. This is attributed to the fact that the dynamics are highly sensitive to the energy fluctuations covered by the width of the excited-state $\Delta E$ distributions (blue histograms and PDFs in Figure 4). Comparison of the average of $150{ }^{1}$ MLCT population curves with the one obtained by the nonequilibrium CPCM Hamiltonian shows only small deviations (Figure 5). This is because the energy shift caused by the solvent relaxation is small, relative to the spread in $\Delta E$ due to the solvent disorder. Therefore, the solvent relaxation does not have a significant impact on the population dynamics of 

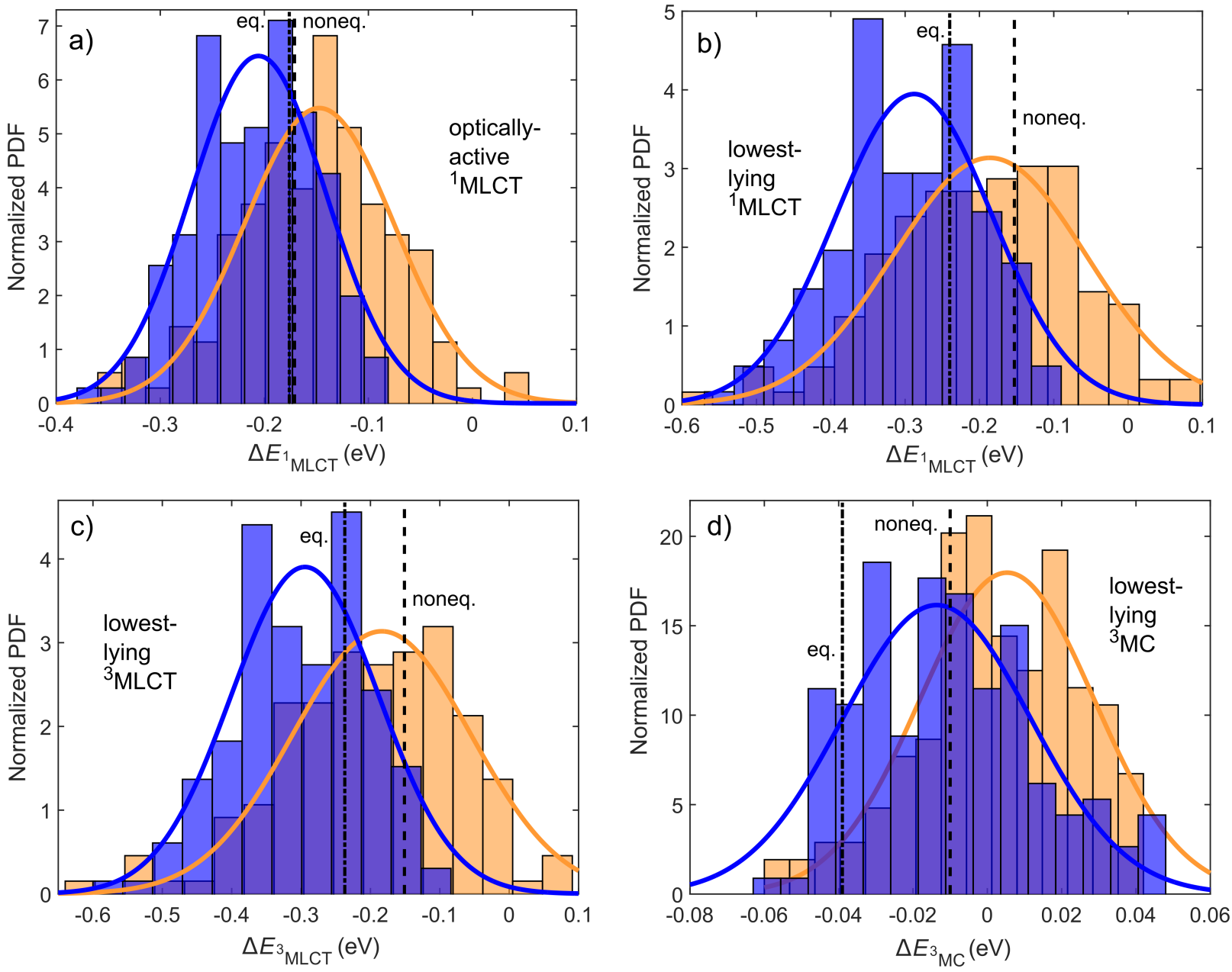

Figure 4: Calculated normalized probability distribution function (PDF) of the energy stabilization of the a) optically-active ${ }^{1} \mathrm{MLCT}$ and the lowest-lying b) $\left.{ }^{1} \mathrm{MLCT}, \mathrm{c}\right){ }^{3} \mathrm{MLCT}$, and d) ${ }^{3} \mathrm{MC}$ states of $\left[\mathrm{Fe}(\mathrm{bmicp})_{2}\right]^{2+}$ by the ACN solvent. The shown histograms were obtained from explicit-solvent QM $\Delta E=E_{\mathrm{sol}}-E_{\mathrm{vac}}$ values, delivered by full explicit-solvent QM TDDFT calculations performed on the solute only (vac) and the system consisting of the solute and explicit solvent molecules (sol). For the latter, solvent configurations were sampled by MD simulations using rigid relaxed solute structures and partial charges corresponding to the ground- (orange) and lowest-lying ${ }^{3}$ MLCT state (blue). The solid lines represent Gaussian fits to the histograms. The vertical dashed lines show the corresponding nonequilibrium (noneq.) and equilibrium (eq.) $\Delta E_{\mathrm{CPCM}}$ values for comparison.

$\mathbf{1}$, and the most important solvent effects are accounted for by the nonequlibrium CPCM Hamiltonian, as is clear from Figure 5. 


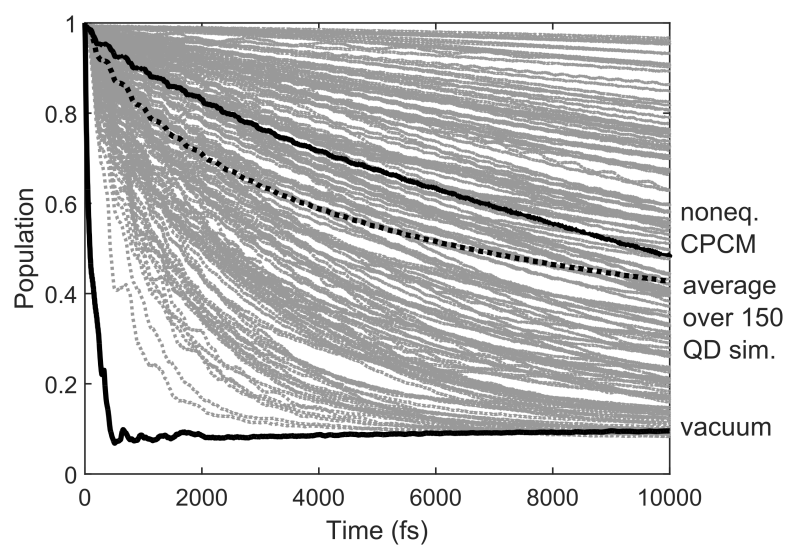

Figure 5: Effect of the solvent relaxation on the overall simulated ${ }^{1}$ MLCT population. The grey curves were obtained from the 150 QD simulations, reflecting different excited-state configurations. For comparison, results obtained from simulations utilizing the gas-phase and QM/CPCM Hamiltonians (heavy solid black lines), as well as the average of the 150 population curves (heavy dotted black line), are shown.

\section{Summary and Conclusion}

In this work, we have investigated the solvent-mediated excited-state dynamics of $\left[\mathrm{Fe}(\text { bmicp })_{2}\right]^{2+}$ using TD-DFT calculations, classical and quantum dynamics simulations. Strikingly, we found that the long-lived excited state of the investigated molecule is not a ${ }^{3} \mathrm{MLCT}$, but the photoexcited ${ }^{1} \mathrm{MLCT}$, which can explain the MLCT lifetime doubling of $\left[\mathrm{Fe}(\mathrm{bmip})_{2}\right]^{2+}$ upon $\mathrm{COOH}$ functionalization. This is because no ultrafast ${ }^{1} \mathrm{MLCT} \rightarrow{ }^{3} \mathrm{MLCT}$ ISC can occur, as all lower-lying ${ }^{3}$ MLCTs are energetically isolated from the optically-active ${ }^{1}$ MLCT state. This observation raises the possibility of electron injection from this ${ }^{1}$ MLCT, when the photoexcited molecule is attached to a semiconductor, such as $\mathrm{TiO}_{2}$. Furthermore, the presented results demonstrate the crucial role of the polar solvent to stabilize the MLCT states; this is accurately described by a polarizable continuum model, in the present case, CPCM. QD simulations using a nonequlibrium QM/CPCM-derived Hamiltonian are found to be a substantial improvement over those based on the Hamiltonian of the isolated molecule; this is highlighted by the fact that the former leads to a long-lived MLCT state (> $10 \mathrm{ps}$ ) and no significant back transfer to the metal during the first few picoseconds, consistently with the experiments. Finally, the solvent relaxation is found to occur on a 
significantly faster timescale $(\sim 0.3-2 \mathrm{ps})$, relative to the population dynamics of the solute. However, this solvent reorganization only slightly modulates the excited-state potentials, leading to small changes in the population dynamics.

\section{Acknowledgement}

The research leading to the presented results has received funding from the People Programme (Marie Curie Actions) of the European Union's Seventh Framework Programme (FP7/ 2007-2013) under REA Grant Agreement No. 609405 (COFUNDPostdocDTU), the Danish Council for Independent Research, Grant No. 4002-00272, the Independent Research Fund Denmark, Grant No. 8021-00347B, and was also supported by the "Lendület" (Momentum) Program of the Hungarian Academy of Sciences (LP2013-59). The authors are grateful for Thomas J. Penfold, Tamás Rozgonyi, and Niels E. Henriksen for fruitful discussions.

\section{Supporting Information Available}

Parameters of the applied spin-vibronic Hamiltonians, TD-DFT excitation energies of 2 at the FC geometry in vacuum and $\mathrm{ACN}$ (noneq.-CPCM), calculated partial atomic charges of 1, computational details of the explicit-solvent QM calculations and QD simulations, results of the out-of-equilibrium MD simulations, and natural transition orbitals (NTOs) corresponding to the singlet excited states computed by QM/CPCM (PDF). Animation of normal modes $\nu_{6}, \nu_{15}, \nu_{35}$, and $\nu_{46}(\mathrm{AVI})$.

This material is available free of charge via the Internet at http://pubs.acs.org/.

\section{References}

(1) Chergui, M. Ultrafast Photophysics of Transition Metal Complexes. Acc. Chem. Res. 2015, 48, 801-808. 
(2) Iwamura, M.; Takeuchi, S.; Tahara, T. Ultrafast Excited-State Dynamics of Copper(I) Complexes. Acc. Chem. Res. 2015, 48, 782-791.

(3) Tavernelli, I. Nonadiabatic Molecular Dynamics Simulations: Synergies between Theory and Experiments. Acc. Chem. Res. 2015, 48, 792-800.

(4) Balzani, V.; Credi, A.; Venturi, M. Photochemical Conversion of Solar Energy. ChemSusChem 2008, 1, 26-58.

(5) Maeda, K.; Domen, K. Photocatalytic Water Splitting: Recent Progress and Future Challenges. J. Phys. Chem. Lett. 2010, 1, 2655-2661.

(6) Ahmad, H.; Kamarudin, S.; Minggu, L.; Kassim, M. Hydrogen from Photo-Catalytic Water Splitting Process: A Review. Renew. Sust. Energ. Rev. 2015, 43, 599 - 610.

(7) Kahn, O.; Martinez, C. J. Spin-Transition Polymers: From Molecular Materials Toward Memory Devices. Science 1998, 279, 44-48.

(8) Natterer, F.; Yang, K.; Paul, W.; Willke, P.; Choi, T.; Greber, T.; Heinrich, A.; Lutz, C. Reading and Writing Single-Atom Magnets. Nature 2017, 543, 226-228.

(9) Liu, L.; Duchanois, T.; Etienne, T.; Monari, A.; Beley, M.; Assfeld, X.; Haacke, S.; Gros, P. C. A New Record Excited State ${ }^{3}$ MLCT Lifetime for Metalorganic Iron(ii) Complexes. Phys. Chem. Chem. Phys. 2016, 18, 12550-12556.

(10) Harlang, T.; Liu, Y.; Gordivska, O.; Fredin, L.; Ponseca, C.; Huang, P.; Chábera, P.; Kjaer, K.; Mateos, H.; Uhlig, J. et al. Iron Sensitizer Converts Light to Electrons with 92\% Yield. Nat. Chem. 2015, 7, 883-889.

(11) Zewail, A. H. Laser Femtochemistry. Science 1988, 242, 1645-1653.

(12) Bargheer, M.; Zhavoronkov, N.; Woerner, M.; Elsaesser, T. Recent Progress in Ultrafast X-ray Diffraction. ChemPhysChem 2006, 7, 783-792. 
(13) Kraus, P. M.; Zürch, M.; Cushing, S. K.; Neumark, D. M. The Ultrafast X-ray Spectroscopic Revolution in Chemical Dynamics. Nat. Rev. 2018, 2, 82-94.

(14) Srinivasan, R.; Lobastov, V.; Ruan, C.-Y.; Zewail, A. Ultrafast Electron Diffraction (UED). Helv. Chim. Acta 2003, 86, 1761-1799.

(15) Crespo-Otero, R.; Barbatti, M. Recent Advances and Perspectives on Nonadiabatic Mixed Quantum-Classical Dynamics. Chem. Rev. 2018, 118, 7026-7068.

(16) Curchod, B. F. E.; Martnez, T. J. Ab Initio Nonadiabatic Quantum Molecular Dynamics. Chem. Rev. 2018, 118, 3305-3336.

(17) Tavernelli, I.; Curchod, B. F.; Rothlisberger, U. Nonadiabatic Molecular Dynamics with Solvent Effects: A LR-TDDFT QM/MM Study of Ruthenium (II) Tris (bipyridine) in Water. Chem. Phys. 2011, 391, $101-109$.

(18) Atkins, A. J.; González, L. Trajectory Surface-Hopping Dynamics Including Intersystem Crossing in $\left[\mathrm{Ru}(\mathrm{bpy})_{3}\right]^{2+}$. J. Phys. Chem. Lett. 2017, 8, 3840-3845.

(19) Capano, G.; Penfold, T. J.; Chergui, M.; Tavernelli, I. Photophysics of a Copper Phenanthroline Elucidated by Trajectory and Wavepacket-Based Quantum Dynamics: A Synergetic Approach. Phys. Chem. Chem. Phys. 2017, 19, 19590-19600.

(20) Capano, G.; Chergui, M.; Rothlisberger, U.; Tavernelli, I.; Penfold, T. J. A Quantum Dynamics Study of the Ultrafast Relaxation in a Prototypical Cu(I)-Phenanthroline. J. Phys. Chem. A 2014, 118, 9861-9869.

(21) Eng, J.; Gourlaouen, C.; Gindensperger, E.; Daniel, C. Spin-Vibronic Quantum Dynamics for Ultrafast Excited-State Processes. Acc. Chem. Res. 2015, 48, 809-817.

(22) Pápai, M.; Vankó, G.; Rozgonyi, T.; Penfold, T. J. High-Efficiency Iron Photosensitizer Explained with Quantum Wavepacket Dynamics. J. Phys. Chem. Lett. 2016, 7, 20092014. 
(23) Pápai, M.; Penfold, T. J.; Møller, K. B. Effect of tert-Butyl Functionalization on the Photoexcited Decay of a Fe(II)-N-Heterocyclic Carbene Complex. J. Phys. Chem. C 2016, 120, 17234-17241.

(24) Fumanal, M.; Gindensperger, E.; Daniel, C. Ultrafast Excited-State Decays in $\left[\operatorname{Re}(\mathrm{CO})_{3}(\mathrm{~N}, \mathrm{~N})(\mathrm{L})\right]^{n+}$ : Nonadiabatic Quantum Dynamics. J. Chem. Theory Comput. 2017, 13, 1293-1306.

(25) Fumanal, M.; Gindensperger, E.; Daniel, C. Ligand Substitution and Conformational Effects on the Ultrafast Luminescent Decay of $[\operatorname{Re}(\mathrm{CO}) 3($ phen $)(\mathrm{L})]+(\mathrm{L}=$ imidazole, pyridine): Non-Adiabatic Quantum Dynamics. Phys. Chem. Chem. Phys. 2018, 20, $1134-1141$.

(26) van der Veen, R. M.; Cannizzo, A.; van Mourik, F.; Vlek, A.; Chergui, M. Vibrational Relaxation and Intersystem Crossing of Binuclear Metal Complexes in Solution. J. Am. Chem. Soc. 2011, 133, 305-315.

(27) Monni, R.; Aubck, G.; Kinschel, D.; Aziz-Lange, K. M.; Gray, H. B.; Vlek, A.; Chergui, M. Conservation of Vibrational Coherence in Ultrafast Electronic Relaxation: The Case of Diplatinum Complexes in Solution. Chem. Phys. Lett. 2017, 683, 112 - 120.

(28) Monni, R.; Capano, G.; Auböck, G.; Gray, H. B.; Vlček, A.; Tavernelli, I.; Chergui, M. Vibrational Coherence Transfer in the Ultrafast Intersystem Crossing of a Diplatinum Complex in Solution. Proc. Natl. Acad. Sci. U. S. A. 2018, 115, E6396-E6403.

(29) Kjær, K. S.; Kunnus, K.; Harlang, T. C. B.; Van Driel, T. B.; Ledbetter, K.; Hartsock, R. W.; Reinhard, M. E.; Koroidov, S.; Li, L.; Laursen, M. G. et al. Solvent Control of Charge Transfer Excited State Relaxation Pathways in $\left[\mathrm{Fe}\left(2,2^{\prime} \text {-bipyridine }\right)(\mathrm{CN})_{4}\right]^{2-}$. Phys. Chem. Chem. Phys. 2018, 20, 4238-4249.

(30) Persico, M.; Granucci, G.; Inglese, S.; Laino, T.; Toniolo, A. Semiclassical Simulation 
of Photochemical Eeactions in Condensed Phase. J. Mol Struct.: THEOCHEM 2003, 621, $119-126$.

(31) Fingerhut, B. P.; Oesterling, S.; Haiser, K.; Heil, K.; Glas, A.; Schreier, W. J.; Zinth, W.; Carell, T.; de Vivie-Riedle, R. ONIOM Approach for Non-Adiabatic Onthe-Fly Molecular Dynamics Demonstrated for the Backbone Controlled Dewar Valence Isomerization. J. Chem. Phys. 2012, 136, 204307.

(32) Cerezo, J.; Liu, Y.; Lin, N.; Zhao, X.; Improta, R.; Santoro, F. Mixed Quantum/Classical Method for Nonadiabatic Quantum Dynamics in Explicit Solvent Models: The $\pi \pi^{*} / n \pi^{*}$ Decay of Thymine in Water as a Test Case. J. Chem. Theory Comput. 2018, 14, 820-832.

(33) Liu, Y.; Harlang, T.; Canton, S. E.; Chábera, P.; Suárez-Alcántara, K.; Fleckhaus, A.; Vithanage, D. A.; Göransson, E.; Corani, A.; Lomoth, R. et al. Towards Longer-Lived Metal-to-Ligand Charge Transfer States of Iron(ii) Complexes: An N-Heterocyclic Carbene Approach. Chem. Commun. 2013, 49, 6412-6414.

(34) Duchanois, T.; Etienne, T.; Cebrián, C.; Liu, L.; Monari, A.; Beley, M.; Assfeld, X.; Haacke, S.; Gros, P. C. An Iron-Based Photosensitizer with Extended Excited-State Lifetime: Photophysical and Photovoltaic Properties. Eur. J. Inorg. Chem. 2015, 2015, 2469-2477.

(35) Cederbaum, L. S.; Köppel, H.; Domcke, W. Multimode Vibronic Coupling Effects in Molecules. Int. J. Quantum Chem. 1981, 20, 251-267.

(36) Köppel, H.; Domcke, W.; Cederbaum, L. S. Advances in Chemical Physics; John Wiley \& Sons, Inc., 1984; Chapter 57, pp 59-246.

(37) Gatti, F.; Lasorne, B.; Meyer, H.-D.; Nauts, A. Applications of Quantum Dynamics in Chemistry, Lecture Notes in Chemistry, vol 98; Springer International Publishing, 2017. 
(38) Penfold, T. J.; Gindensperger, E.; Daniel, C.; Marian, C. M. Spin-Vibronic Mechanism for Intersystem Crossing. Chem. Rev. 2018, 118, 6975-7025.

(39) Reiher, M.; Salomon, O.; Artur Hess, B. Reparameterization of Hybrid Functionals Based on Energy Differences of States of Different Multiplicity. Theor. Chem. Acc. 2001, 10\%, 48-55.

(40) Schäfer, A.; Huber, C.; Ahlrichs, R. Fully Optimized Contracted Gaussian Basis Sets of Triple Zeta Valence Quality for Atoms Li to Kr. J. Chem. Phys. 1994, 100, 5829-5835.

(41) Daku, L. M. L.; Vargas, A.; Hauser, A.; Fouqueau, A.; Casida, M. E. Assessment of Density Functionals for the High-Spin/Low-Spin Energy Difference in the Low-Spin Iron(II) Tris(2,2?-bipyridine) Complex. Chem. Phys. Chem. 2005, 6, 1393-1410.

(42) Pápai, M.; Vankó, G.; de Graaf, C.; Rozgonyi, T. Theoretical Investigation of the Electronic Structure of Fe(II) Complexes at Spin-State Transitions. J. Chem. Theory Comput. 2013, 9, 509-519.

(43) Leshchev, D.; Harlang, T.; Fredin, L.; Khakhulin, D.; Liu, Y.; Biasin, E.; Laursen, M.; Newby, G.; Haldrup, K.; Nielsen, M. et al. Tracking the Picosecond Deactivation Dynamics of a Photoexcited Iron Carbene Complex by Time-Resolved X-ray Scattering. Chem. Sci. 2018, 9, 405-414, cited By 0.

(44) Barone, V.; Cossi, M. Quantum Calculation of Molecular Energies and Energy Gradients in Solution by a Conductor Solvent Model. J. Phys. Chem. A 1998, 102, 19952001.

(45) Aguilar, M. A.; Olivares del Valle, F. J.; Tomasi, J. Nonequilibrium Solvation: An Ab Initio Quantum-Mechanical Method in the Continuum Cavity Model Approximation. J. Chem. Phys. 1993, 98, 7375-7384. 
(46) De Vetta, M.; Menger, M. F. S. J.; Nogueira, J. J.; González, L. Solvent Effects on Electronically Excited States: QM/Continuum Versus QM/Explicit Models. J. Phys. Chem. B 2018, 122, 2975-2984.

(47) Nogueira, J. J.; González, L. Computational Photophysics in the Presence of an Environment. Annu. Rev. Phys. Chem. 2018, 69, 473-497.

(48) Cossi, M.; Barone, V. Time-Dependent Density Functional Theory for Molecules in Liquid Solutions. J. Chem. Phys. 2001, 115, 4708-4717.

(49) Frisch, M. J.; Trucks, G. W.; Schlegel, H. B.; Scuseria, G. E.; Robb, M. A.; Cheeseman, J. R.; Scalmani, G.; Barone, V.; Petersson, G. A.; Nakatsuji, H. et al. Gaussian 09 Revision D.01. 2016.

(50) Wang, F.; Ziegler, T. A Simplified Relativistic Time-Dependent Density-Functional Theory Formalism for the Calculations of Excitation Energies Including Spin-Orbit Coupling Effect. J. Chem. Phys. 2005, 123, 154102.

(51) van Lenthe, E.; Baerends, E. J.; Snijders, J. G. Relativistic Regular Two-Component Hamiltonians. J. Chem. Phys. 1993, 99, 4597-4610.

(52) Klamt, A.; Schrmann, G. COSMO: A New Approach to Dielectric Screening in Solvents with Explicit Expressions for the Screening Energy and its Gradient. J. Chem. Soc., Perkin Trans. 2 1993, 799-805.

(53) te Velde, G.; Bickelhaupt, F. M.; Baerends, E. J.; Fonseca Guerra, C.; van Gisbergen, S. J. A.; Snijders, J. G.; Ziegler, T. Chemistry with ADF. J. Comput. Chem. 2001, 22, $931-967$.

(54) Meyer, H.-D.; Manthe, U.; Cederbaum, L. The Multi-Configurational Time-Dependent Hartree Approach. Chem. Phys. Lett. 1990, 165, 73 - 78. 
(55) Beck, M.; Jäckle, A.; Worth, G.; Meyer, H.-D. The Multiconfiguration Time-Dependent Hartree (MCTDH) Method: A Highly Efficient Algorithm for Propagating Wavepackets. Phys. Rep. 2000, 324, $1-105$.

(56) Humphreys, D. D.; Friesner, R. A.; Berne, B. J. A Multiple-Time-Step Molecular Dynamics Algorithm for Macromolecules. J. Phys. Chem. 1994, 98, 6885-6892.

(57) Bowers, K. J.; Chow, E.; Xu, H.; Dror, R. O.; Eastwood, M. P.; Gregersen, B. A.; Klepeis, J. L.; Kolossvary, I.; Moraes, M. A.; Sacerdoti, F. D. et al. Scalable Algorithms for Molecular Dynamics Simulations on Commodity Clusters. Proceedings of the 2006 ACM/IEEE Conference on Supercomputing. New York, NY, USA, 2006.

(58) Jorgensen, W. L.; Maxwell, D. S.; Tirado-Rives, J. Development and Testing of the OPLS All-Atom Force Field on Conformational Energetics and Properties of Organic Liquids. J. Am. Chem. Soc. 1996, 118, 11225-11236.

(59) Guàrdia, E.; Pinzón, R.; Casulleras, J.; Orozco, M.; Luque, F. J. Comparison of Different Three-Site Interaction Potentials for Liquid Acetonitrile. Mol. Simul. 2001, 26, $287-306$.

(60) Abedi, M; Levi, G.; Zederkof, D. B.; Henriksen, N. E; Pápai, M.; Møller, K. B. Excited-State Solvation Structure of Transition Metal Complexes from Molecular Dynamics Simulations and Assessment of Partial Atomic Charge Methods. 2018, arXiv:1812.09090.

(61) Breneman, C. M.; Wiberg, K. B. Determining Atom-Centered Monopoles from Molecular Electrostatic Potentials. The Need for High Sampling Density in Formamide Conformational Analysis. J. Comput. Chem. 1990, 11, 361-373.

(62) Berendsen, H. J. C.; Postma, J. P. M.; van Gunsteren, W. F.; DiNola, A.; Haak, J. R. Molecular Dynamics with Coupling to an External Bath. J. Chem. Phys. 1984, 81, $3684-3690$. 
(63) Evans, D. J.; Holian, B. L. The Nose-Hoover Thermostat. J. Chem. Phys. 1985, 83, 4069-4074.

(64) Fredin, L. A.; Wärnmark, K.; Sundström, V.; Persson, P. Molecular and Interfacial Calculations of Iron(II) Light Harvesters. ChemSusChem 2016, 9, 667-675.

(65) Gawelda, W.; Cannizzo, A.; Pham, V.-T.; van Mourik, F.; Bressler, C.; Chergui, M. Ultrafast Nonadiabatic Dynamics of $[\mathrm{FeII}(\mathrm{bpy}) 3] 2+$ in Solution. J. Am. Chem. Soc. 2007, 129, 8199-8206.

(66) Cannizzo, A.; Milne, C.; Consani, C.; Gawelda, W.; Bressler, C.; van Mourik, F.; Chergui, M. Light-Induced Spin Crossover in Fe(II)-Based Complexes: The Full Photocycle Unraveled by Ultrafast Optical and X-ray Spectroscopies. Coord. Chem. Rev. 2010, $254,2677-2686$.

(67) Sousa, C.; de Graaf, C.; Rudavskyi, A.; Broer, R.; Tatchen, J.; Etinski, M.; Marian, C. M. Ultrafast Deactivation Mechanism of the Excited Singlet in the Light-Induced Spin Crossover of [Fe(2,2'-bipyridine)3]2+. Chem. Eur. J. 2013, 19, 17541-17551.

(68) Liu, Y.; Persson, P.; Sundström, V.; Wärnmark, K. Fe N-Heterocyclic Carbene Complexes as Promising Photosensitizers. Acc. Chem. Res. 2016, 49, 1477-1485.

(69) van Driel, T.; Kjær, K.; Hartsock, R.; Dohn, A.; Harlang, T.; Chollet, M.; Christensen, M.; Gawelda, W.; Henriksen, N.; Kim, J. et al. Atomistic Characterization of the Active-Site Solvation Dynamics of a Model Photocatalyst. Nat. Commun. 2016, 7.

(70) Levi, G.; Pápai, M.; Henriksen, N. E.; Dohn, A. O.; Møller, K. B. Solution Structure and Ultrafast Vibrational Relaxation of the PtPOP Complex Revealed by SCF-QM/MM Direct Dynamics Simulations. J. Phys.Chem. C 2018, 122, 7100-7119.

(71) Improta, R.; Barone, V.; Scalmani, G.; Frisch, M. J. A State-Specific Polarizable Con- 
tinuum Model Time Dependent Density Functional Theory Method for Excited State Calculations in Solution. J. Chem. Phys. 2006, 125, 054103. 


\section{TOC Graphic}

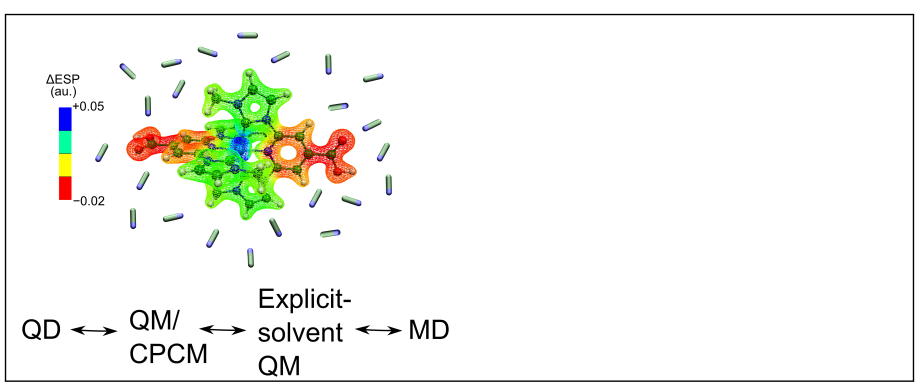

